CRYSTALLOGRAPHIC COMMUNICATIONS

ISSN 2056-9890

Received 22 January 2020

Accepted 10 February 2020

Edited by C. Rizzoli, Universita degli Studi di Parma, Italy

Keywords: crystal structure; charge assisted hydrogen bonding; thiazolidine ring; envelope conformation; Hirshfeld surface analysis.

CCDC reference: 1837123

Supporting information: this article has supporting information at journals.iucr.org/e

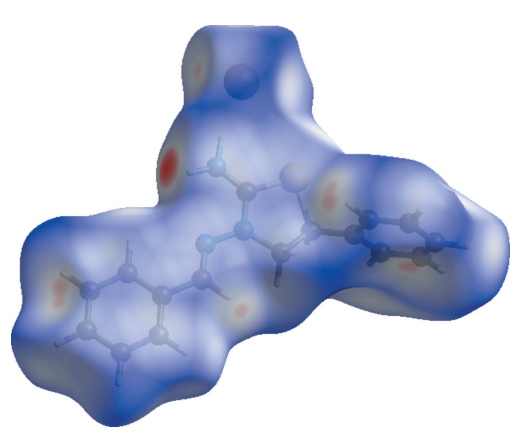

OPEN $\odot$ ACCESS

\section{Crystal structure and Hirshfeld surface analysis of (E)-3-(benzylideneamino)-5-phenylthiazolidin-2- iminium bromide}

\author{
Gulnara Sh. Duruskari, ${ }^{a}$ Mehmet Akkurt, ${ }^{\mathrm{b}}$ Gunay Z. Mammadova, ${ }^{\mathrm{a}}$ Taras Chyrka ${ }^{\mathrm{c} *}$ \\ and Abel M. Maharramov ${ }^{a}$
}

\begin{abstract}
a Organic Chemistry Department, Baku State University, Z. Xalilov str. 23, Az, 1148 Baku, Azerbaijan, ${ }^{\mathbf{b}}$ Department of Physics, Faculty of Sciences, Erciyes University, 38039 Kayseri, Turkey, and ${ }^{\mathrm{C} D e p a r t m e n t}$ of Theoretical and Industrial Heat Engineering (TPT), National Technical University of Ukraine "Igor Sikorsky Kyiv Polytechnic Institute", 03056, Kyiv, Ukraine. *Correspondence e-mail: mustford@ukr.net
\end{abstract}

The central thiazolidine ring of the title salt, $\mathrm{C}_{16} \mathrm{H}_{16} \mathrm{~N}_{3} \mathrm{~S}^{+} \cdot \mathrm{Br}^{-}$, adopts an envelope conformation, with the $\mathrm{C}$ atom bearing the phenyl ring as the flap atom. In the crystal, the cations and anions are linked by $\mathrm{N}-\mathrm{H} \cdots \mathrm{Br}$ hydrogen bonds, forming chains parallel to the $b$-axis direction. Hirshfeld surface analysis and two-dimensional fingerprint plots indicate that the most important contributions to the crystal packing are from $\mathrm{H} \cdots \mathrm{H}(46.4 \%), \mathrm{C} \cdots \mathrm{H} / \mathrm{H} \cdots \mathrm{C}$ $(18.6 \%)$ and $\mathrm{H} \cdots \mathrm{Br} / \mathrm{Br} \cdots \mathrm{H}(17.5 \%)$ interactions.

\section{Chemical context}

Sulfur and nitrogen-containing heterocycles maintain their importance as key fragments of drugs and medicinally active compounds (Pathania et al., 2019). Moreover, azomethinecontaining structural motifs have been widely employed for industrial purposes as they exhibit a broad range of biological activities, and are used in synthesis, catalysis and the design of materials (Gurbanov et al., 2017, 2018; Mahmoudi et al., 2018a,b,c; Mamedov et al., 2018). Nowadays, N-ligands are key players in a wide diversity of fields, namely in coordination, metal-organic, pharmaceutical and medicinal chemistry, biologically active compounds, catalysis, non-covalent interactions and supramolecular assemblies (Maharramov et al., 2011, 2018; Mahmudov et al., 2013, 2014, 2017a,b, 2019; Mamedov et al., 2015). In our previous studies we have reported on the molecular structural properties of a series of 5-phenylthiazolidin-2-imine derivatives (Akkurt et al., 2018a,b; Duruskari et al., 2019a,b; Khalilov et al., 2019; Maharramov et al., 2019). Following further study in this field, herein we report the crystal structure and Hirshfeld surface analysis of the title compound, (E)-3-(benzylideneamino)-5phenylthiazolidin-2-iminium bromide.

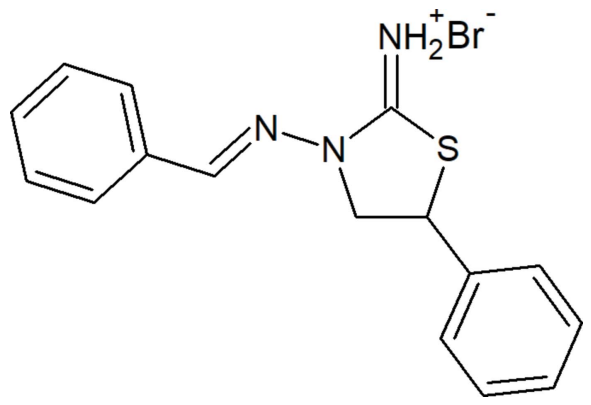


Table 1

Hydrogen-bond geometry $\left(\AA,^{\circ}\right)$.

\begin{tabular}{lllll}
\hline$D-\mathrm{H} \cdots A$ & $D-\mathrm{H}$ & $\mathrm{H} \cdots A$ & $D \cdots A$ & $D-\mathrm{H} \cdots A$ \\
\hline $\mathrm{N} 3-\mathrm{H} 3 A \cdots \mathrm{Br} 1$ & 0.90 & 2.37 & $3.258(3)$ & 168 \\
$\mathrm{~N} 3-\mathrm{H} 3 B \cdots \mathrm{Br} 1^{\mathrm{i}}$ & 0.90 & 2.55 & $3.399(3)$ & 158 \\
\hline
\end{tabular}

Symmetry code: (i) $-x+1, y+\frac{1}{2},-z+\frac{3}{2}$.

\section{Structural commentary}

The thiazolidine ring $(\mathrm{S} 1 / \mathrm{N} 2 / \mathrm{C} 1-\mathrm{C} 3)$ in the cation of the title salt (Fig. 1) adopts an envelope conformation, with the $\mathrm{C}$ atom bearing the phenyl ring as the flap atom; the puckering parameters are $Q(2)=0.318(3) \AA$ and $\varphi(2)=42.0(5)^{\circ}$. The mean plane of the thiazolidine ring makes dihedral angles of $18.28(15)$ and $83.19(15)^{\circ}$, respectively, with the C5-C10 and C11-C16 phenyl rings of the 3-(benzylideneamino) and 5phenylthiazolidin groups, while the dihedral angle between them is $82.54(15)^{\circ}$. The torsion angle of the $\mathrm{N} 2-\mathrm{N} 1-\mathrm{C} 4-\mathrm{C} 5$ bridge that links the thiazolidine and 3-(benzylideneamino) units is $-175.7(3)^{\circ}$.

\section{Supramolecular features}

In the crystal, adjacent cations and anions are linked by pairs of $\mathrm{N}-\mathrm{H} \cdots \mathrm{Br}$ hydrogen bonds (Table 1, Fig. 2), forming chains running parallel to the $b$-axis direction. $\mathrm{C}-\mathrm{H} \cdots \pi$ interactions or $\pi-\pi$ stacking interactions contributing to the stabilization of the crystal packing are not observed.

\section{Hirshfeld surface analysis}

The Hirshfeld surface analysis (Spackman \& Jayatilaka, 2009) of the title compound was generated by CrystalExplorer 3.1 (Wolff et al., 2012), and comprises $d_{\text {norm }}$ surface plots and twodimensional fingerprint plots (Spackman \& McKinnon, 2002). A $d_{\text {norm }}$ surface plot of the title compound mapped over $d_{\text {norm }}$

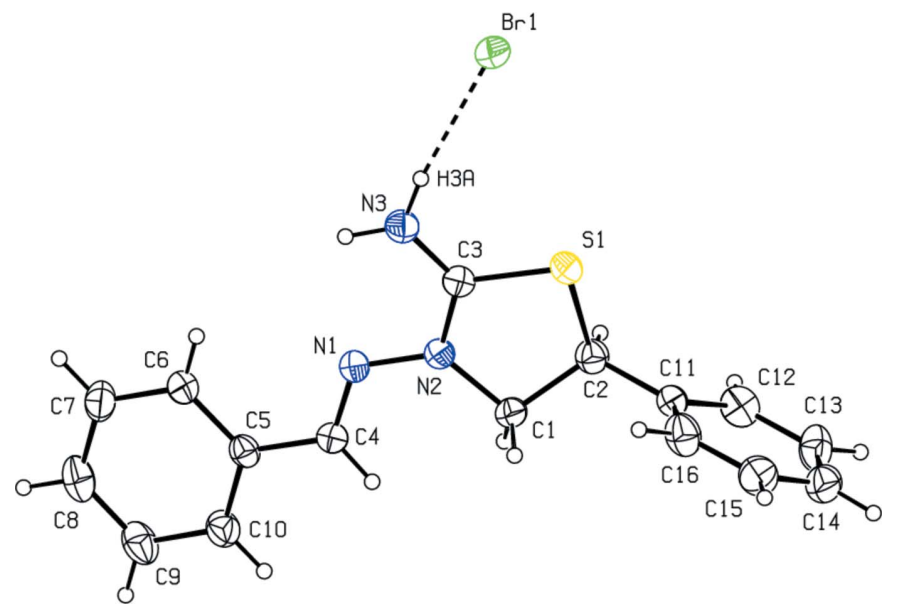

Figure 1

The molecular structure of the title salt, with the atom labelling. Displacement ellipsoids are drawn at the $30 \%$ probability level. The interionic hydrogen bond is shown as a dashed line.

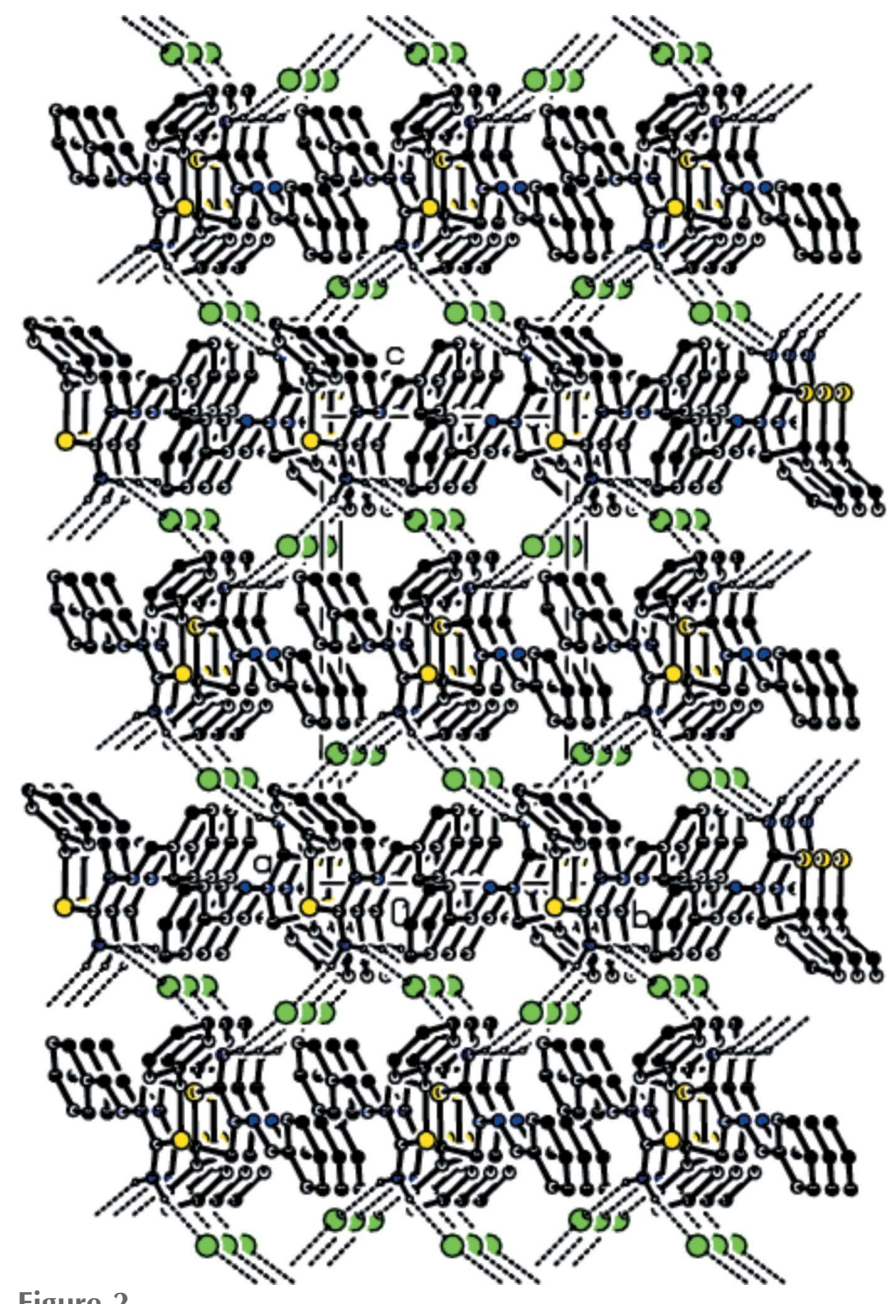

Figure 2

A view of the crystal packing showing the formation of chains parallel to the $b$ axis through $\mathrm{N}-\mathrm{H} \cdots \mathrm{Br}$ hydrogen bonds (dashed lines).

using a standard surface resolution with a fixed colour scale of -0.3485 (red) to 1.3503 a.u. (blue) is shown in Fig. 3. The darkred spots on the $d_{\text {norm }}$ surface arise as a result of short inter-

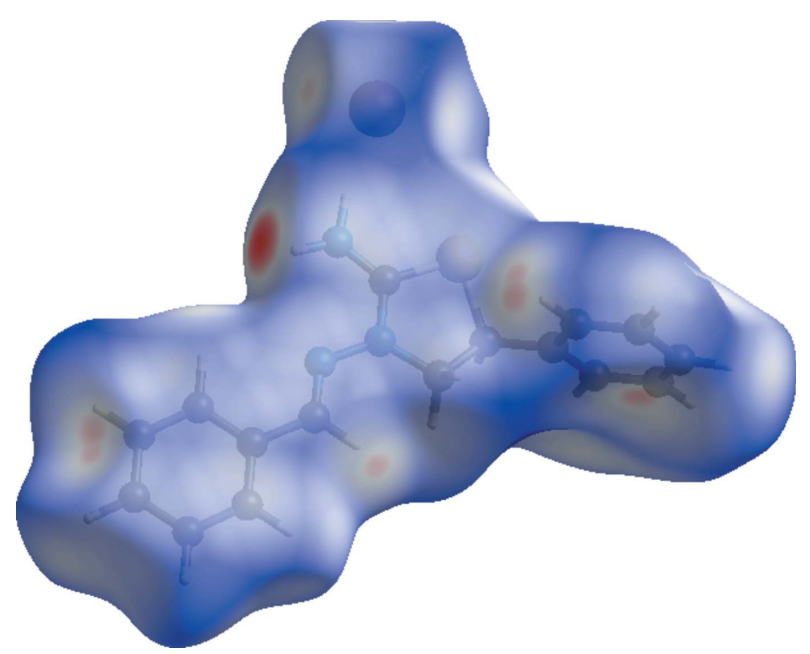

Figure 3

View of the three-dimensional Hirshfeld surface of the title salt plotted over $d_{\text {norm }}$. 
Table 2

Summary of short interatomic contacts $(\AA)$ in the title salt.

\begin{tabular}{lll}
\hline Contact & Distance & Symmetry operation \\
\hline $\mathrm{Br} 1 \cdots \mathrm{H} 3 A(\mathrm{~N} 3)$ & 2.37 & $x, y, z$ \\
$\mathrm{Br} 1 \cdots \mathrm{H} 3 B(\mathrm{~N} 3)$ & 2.55 & $1-x,-\frac{1}{2}+y, \frac{3}{2}-z$ \\
$\mathrm{Br} 1 \cdots \mathrm{H} 14 A(\mathrm{C} 14)$ & 3.14 & $-x, 1-y, 1-z$ \\
$\mathrm{Br} 1 \cdots \mathrm{H} 4 A(\mathrm{C} 4)$ & 2.96 & $x, \frac{3}{2}-y, \frac{1}{2}+z$ \\
$\mathrm{Br} 1 \cdots \mathrm{H} 12 A(\mathrm{C} 12)$ & 3.02 & $x, \frac{1}{2}-y, \frac{1}{2}+z$ \\
\hline
\end{tabular}

atomic contacts (Table 2), while the other weaker intermolecular interactions appear as light-red spots.

The shape index of the Hirshfeld surface is a tool to visualize $\pi-\cdot \pi$ stacking interactions by the presence of adjacent red and blue triangles; if there are no adjacent red and/or blue triangles, then there are no $\pi-\pi$ interactions. Fig. 4 clearly suggests that there are no $\pi-\pi$ interactions present in the title compound. Fig. 5( $a$ ) shows the two-dimensional fingerprint of the sum of the contacts contributing to the Hirshfeld surface represented in normal mode (Tables 1 and 2). The fingerprint plots delineated into $\mathrm{H} \cdots \mathrm{H}(46.4 \%), \mathrm{C} \cdots \mathrm{H} / \mathrm{H} \cdots \mathrm{C}(18.6 \%)$, $\mathrm{H} \cdots \mathrm{Br} / \mathrm{Br} \cdots \mathrm{H}(17.5 \%), \mathrm{H} \cdots \mathrm{S} / \mathrm{S} \cdots \mathrm{H}(4.5 \%)$ and $\mathrm{C} \cdots \mathrm{N} /$ N. .C $(3.7 \%)$ contacts are shown in Fig. $5 b-f$.

The most significant intermolecular interactions are the $\mathrm{H} \cdot \mathrm{H}$ interactions (46.4\%) (Fig. 5b). All of the contributions to the Hirshfeld surface are given in Table 3 . The large number of $\mathrm{H} \cdots \mathrm{H}, \mathrm{C} \cdots \mathrm{H} / \mathrm{H} \cdots \mathrm{C}$ and $\mathrm{H} \cdots \mathrm{Br} / \mathrm{Br} \cdots \mathrm{H}$ interactions suggest that van der Waals interactions and hydrogen bonding play the major roles in the crystal packing (Hathwar et al., 2015).

\section{Database survey}

A search of the Cambridge Structural Database (CSD, Version 5.40, February 2019; Groom et al., 2016) for 2-thiazolidiniminium compounds gave ten hits, viz. MOJGUQ (Duruskari et al., 2019a), XOWXAL (Duruskari et al., 2019b), BOBWIB (Khalilov et al., 2019), UDELUN (Akkurt et al., 2018a), WILBIC (Marthi et al., 1994), WILBOI (Marthi et al.,

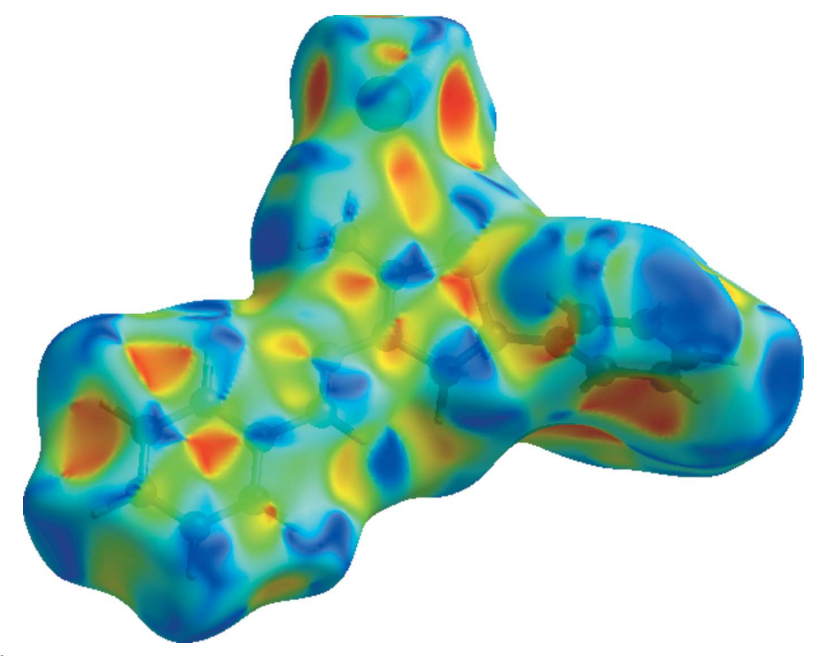

Figure 4

Hirshfeld surface of the title salt plotted over shape-index.
Table 3

Percentage contributions of interatomic contacts to the Hirshfeld surface for the title salt.

\begin{tabular}{ll}
\hline Contact & Percentage contribution \\
\hline $\mathrm{H} \cdots \mathrm{H}$ & 46.4 \\
$\mathrm{C} \cdots \mathrm{H} / \mathrm{H} \cdots \mathrm{C}$ & 18.6 \\
$\mathrm{H} \cdots \mathrm{Br} / \mathrm{Br} \cdots \mathrm{H}$ & 17.5 \\
$\mathrm{H} \cdots \mathrm{S} / \mathrm{S} \cdots \mathrm{H}$ & 4.5 \\
$\mathrm{C} \cdots \mathrm{N} / \mathrm{N} \cdots \mathrm{C}$ & 3.7 \\
$\mathrm{C} \cdots \mathrm{S} / \mathrm{S} \cdots \mathrm{C}$ & 3.0 \\
$\mathrm{H} \cdots \mathrm{N} / \mathrm{N} \cdots \mathrm{H}$ & 2.6 \\
$\mathrm{C} \cdots \mathrm{C}$ & 2.3 \\
$\mathrm{C} \cdots \mathrm{Br} / \mathrm{Br} \cdots \mathrm{C}$ & 0.9 \\
$\mathrm{~N} \cdots \mathrm{S} / \mathrm{S} \cdots \mathrm{N}$ & 0.5 \\
$\mathrm{~N} \cdots \mathrm{N}$ & 0.2 \\
\hline
\end{tabular}

1994), WILBOI01 (Marthi et al., 1994), YITCEJ (Martem'yanova et al., 1993a), YITCAF (Martem'yanova et $a l ., 1993 b)$ and YOPLUK (Marthi et al., 1995).

In the crystal of MOJGUQ (Duruskari et al., 2019a), centrosymmetrically related cations and anions are linked into dimeric units via $\mathrm{N}--\mathrm{H} \cdot \mathrm{Br}$ hydrogen bonds, which are further connected by weak $\mathrm{C}-\mathrm{H} \cdots \mathrm{Br}$ contacts into chains parallel to the $a$ axis. Furthermore, $\mathrm{C}-\mathrm{H} \cdots \pi$ interactions and $\pi-\pi$ stacking interactions [centroid-to-centroid distance $=$ 3.897 (2) $\AA$ ] between the major components of the disordered phenyl ring contribute to the stabilization of the molecular packing. In the crystal of XOWXAL (Duruskari et al., 2019b), the thiazolidine ring adopts an envelope conformation. $\mathrm{N}-$ $\mathrm{H} \cdots \mathrm{Br}$ hydrogen bonds link the components into a threedimensional network. Weak $\pi-\pi$ stacking interactions
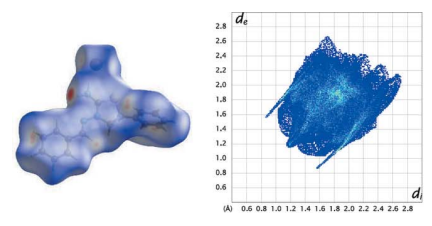

(a) All
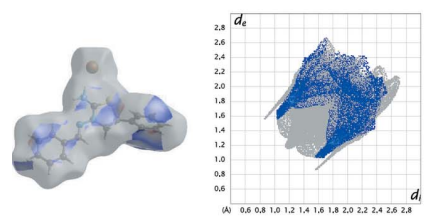

(c) $\mathrm{C} \cdots \mathrm{H} / \mathrm{H} \cdots \mathrm{C}$

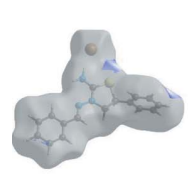

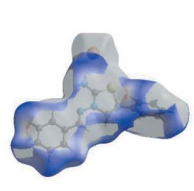

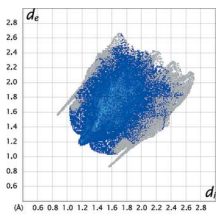

(b) $\mathrm{H} \cdots \mathrm{H}$
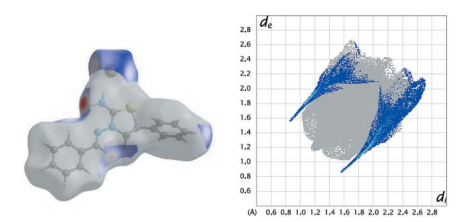

(d) $\mathrm{H} \cdots \mathrm{Br} / \mathrm{Br} \cdots \mathrm{H}$
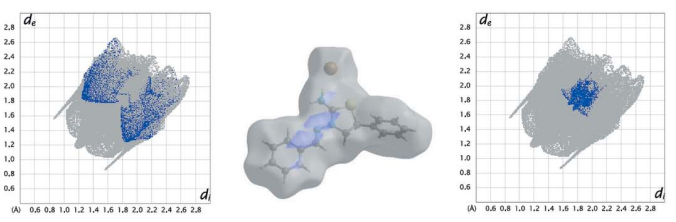

(e) $\mathrm{H} \cdots \mathrm{S} / \mathrm{S} \cdots \mathrm{H}$

(f) $\mathrm{C} \cdots \mathrm{N} / \mathrm{N} \cdots \mathrm{C}$

Figure 5

The Hirshfeld surface representations and the full two-dimensional fingerprint plots for the title salt, showing $(a)$ all interactions, and delineated into $(b) \mathrm{H} \cdots \mathrm{H},(c) \mathrm{C} \cdots \mathrm{H} / \mathrm{H} \cdots \mathrm{C},(d) \mathrm{H} \cdots \mathrm{Br} / \mathrm{Br} \cdots \mathrm{H},(e)$ $\mathrm{H} \cdots \mathrm{S} / \mathrm{S} \cdots \mathrm{H}$ and $(f) \mathrm{C} \cdots \mathrm{N} / \mathrm{N} \cdots \mathrm{C}$ interactions. The $d_{\mathrm{i}}$ and $d_{\mathrm{e}}$ values are the closest internal and external distances (in $\AA$ ) from given points on the Hirshfeld surface. 
Table 4

Experimental details.

\begin{tabular}{|c|c|}
\hline \multicolumn{2}{|l|}{ Crystal data } \\
\hline Chemical formula & $\mathrm{C}_{16} \mathrm{H}_{16} \mathrm{~N}_{3} \mathrm{~S}^{+} \cdot \mathrm{Br}^{-}$ \\
\hline$M_{\mathrm{r}}$ & 362.29 \\
\hline Crystal system, space group & Monoclinic, $P 2_{1} / c$ \\
\hline Temperature $(\mathrm{K})$ & 296 \\
\hline$a, b, c(\AA)$ & $12.138(8), 8.336(5), 15.872(9)$ \\
\hline$\beta\left(^{\circ}\right)$ & $93.910(16)$ \\
\hline$V\left(\AA^{3}\right)$ & $1602.3(17)$ \\
\hline$Z$ & 4 \\
\hline Radiation type & Мо $K \alpha$ \\
\hline$\mu\left(\mathrm{mm}^{-1}\right)$ & 2.69 \\
\hline Crystal size (mm) & $0.21 \times 0.18 \times 0.13$ \\
\hline \multicolumn{2}{|l|}{ Data collection } \\
\hline Diffractometer & Bruker APEXII CCD \\
\hline Absorption correction & $\begin{array}{l}\text { Multi-scan ( } S A D A B S \text {; Bruker, } \\
\text { 2003) }\end{array}$ \\
\hline$T_{\min }, T_{\max }$ & $0.582,0.713$ \\
\hline $\begin{array}{l}\text { No. of measured, independent and } \\
\text { observed }[I>2 \sigma(I)] \text { reflections }\end{array}$ & $23979,3314,2742$ \\
\hline$R_{\text {int }}$ & 0.049 \\
\hline$(\sin \theta / \lambda)_{\max }\left(\AA^{-1}\right)$ & 0.629 \\
\hline \multicolumn{2}{|l|}{ Refinement } \\
\hline$R\left[F^{2}>2 \sigma\left(F^{2}\right)\right], w R\left(F^{2}\right), S$ & $0.040,0.111,1.06$ \\
\hline No. of reflections & 3314 \\
\hline No. of parameters & 190 \\
\hline No. of restraints & 12 \\
\hline $\mathrm{H}$-atom treatment & $\mathrm{H}$-atom parameters constrained \\
\hline$\Delta \rho_{\max }, \Delta \rho_{\min }\left(\mathrm{e} \AA^{-3}\right)$ & $0.74,-0.60$ \\
\hline
\end{tabular}

Computer programs: APEX2 and SAINT (Bruker, 2003), SHELXT2014 (Sheldrick, 2015a), SHELXL2016 (Sheldrick, 2015b) and SHELXTL (Sheldrick, 2008).

between the phenyl rings of adjacent cations also contribute to the molecular packing. In the crystal of BOBWIB (Khalilov et al., 2019), the central thiazolidine ring adopts an envelope conformation. In the crystal, centrosymmetrically related cations and anions are linked into dimeric units via $\mathrm{N}-\mathrm{H} \cdots \mathrm{Br}$ hydrogen bonds, which are further connected by weak $\mathrm{C}-$ $\mathrm{H} \cdots \mathrm{Br}$ hydrogen bonds into chains parallel to [110]. In the crystal of UDELUN (Akkurt et al., 2018a), C-H..Br and $\mathrm{N}-\mathrm{H} \cdots \mathrm{Br}$ hydrogen bonds link the components into a threedimensional network with the cations and anions stacked along the $b$-axis direction. Weak $\mathrm{C}-\mathrm{H} \cdots \pi$ interactions, which only involve the minor disorder component of the ring, also contribute to the molecular packing. In addition, there are also inversion-related $\mathrm{Cl} \cdots \mathrm{Cl}$ halogen bonds and $\mathrm{C}-\mathrm{Cl} \cdots \pi$ (ring) contacts. In the other structures, the $3-\mathrm{N}$ atom carries a $\mathrm{C}-$ substituent instead of an $\mathrm{N}$-substituent as found in the title compound. Three of them were determined to be racemic (WILBIC; Marthi et al., 1994) and two optically active samples (WILBOI and WILBOI01; Marthi et al., 1994) of 3-(2'-chloro2 '-phenylethyl)-2-thiazolidiniminium $p$-toluenesulfonate. In all three structures, the most disordered fragment is the asymmetric $\mathrm{C}$ atom and the $\mathrm{Cl}$ atom attached to it. The disorder of the cation in the racemate corresponds to the presence of both enantiomers at each site in the ratio 0.821 (3):0.179 (3). The system of hydrogen bonds connecting two cations and two anions into 12-membered rings is identical in the racemic and in the optically active crystals. YITCEJ (Martem'yanova et al., 1993a), is a product of the interaction of 2-amino-5-methylthiazoline with methyl iodide, with alkylation at the endocylic nitrogen atom, while YITCAF (Martem'yanova et al., 1993b) is a product of the reaction of 3nitro-5-methoxy-, 3-nitro-5-chloro-, and 3-bromo-5-nitrosalicylaldehyde with the heterocyclic base to form the salt-like complexes.

\section{Synthesis and crystallization}

To the solution of 3-amino-5-phenylthiazolidin-2-iminium bromide $(1 \mathrm{mmol})$ in $20 \mathrm{~mL}$ of ethanol was added benzaldehyde $(1 \mathrm{mmol})$ and the mixture was refluxed for $2 \mathrm{~h}$. After cooling down to room temperature, the reaction product precipitated as colourless single crystals, which were collected by filtration and washed with cold acetone (yield 76\%), m.p. 519 K. Analysis calculated for $\mathrm{C}_{16} \mathrm{H}_{16} \mathrm{BrN}_{3} \mathrm{~S}\left(M_{\mathrm{r}}=362.29\right)$ : $\mathrm{C}$, 53.04; H, 4.45; N, 11.60. Found: C, 53.01; H, 4.42; N, 11.56\%. ${ }^{1} \mathrm{H}$ NMR (300 MHz, DMSO- $\left.d_{6}\right): 4.58\left(k, 1 \mathrm{H}, \mathrm{CH}_{2},{ }^{3} J_{\mathrm{H}-\mathrm{H}}=6.9\right)$; $4,89\left(t, 1 \mathrm{H}, \mathrm{CH}_{2},{ }^{3} J_{\mathrm{H}-\mathrm{H}}=8.1\right) ; 5.60\left(t, 1 \mathrm{H}, \mathrm{CH}-\mathrm{Ar},{ }^{3} J_{\mathrm{H}-\mathrm{H}}=7.5\right)$; 7.37-8.07 ( $m, 10 \mathrm{H}, 10 \mathrm{Ar}-\mathrm{H}) ; 8.44(s, 1 \mathrm{H}, \mathrm{CH}=), 10.35(s, 2 \mathrm{H}$, $\mathrm{NH}=) .{ }^{13} \mathrm{C}$ NMR $\left(75 \mathrm{MHz}\right.$, DMSO- $\left.d_{6}\right): 45.36,55.91,127.76$, 128.65, 128.82, 128.86, 129.09, 131.54, 132.85, 137.48, 151.11, 167.84. MS (ESI), $m / z: 282.30\left[\mathrm{C}_{16} \mathrm{H}_{16} \mathrm{~N}_{3} \mathrm{~S}\right]^{+}$and $79.88 \mathrm{Br}^{-}$.

\section{Refinement}

Crystal data, data collection and structure refinement details are summarized in Table 4. All $\mathrm{H}$ atoms were placed at calculated positions $(\mathrm{N}-\mathrm{H}=0.90 \AA$ and $\mathrm{C}-\mathrm{H}=0.93-0.98 \AA$ ) and refined using a riding model, with $U_{\text {iso }}(\mathrm{H})=1.2 U_{\text {eq }}(\mathrm{N}, \mathrm{C})$. The distances between the carbon atoms of two phenyl groups were constrained with a DFIX instruction [DFIX 1.40 $0.02 \mathrm{C} \mathrm{C}]$.

\section{References}

Akkurt, M., Duruskari, G. S., Toze, F. A. A., Khalilov, A. N. \& Huseynova, A. T. (2018a). Acta Cryst. E74, 1168-1172.

Akkurt, M., Maharramov, A. M., Duruskari, G. S., Toze, F. A. A. \& Khalilov, A. N. (2018b). Acta Cryst. E74, 1290-1294.

Bruker (2003). APEX2, SAINT and SADABS. Bruker AXS Inc., Madison, Wisconsin, USA.

Duruskari, G. S., Khalilov, A. N., Akkurt, M., Mammadova, G. Z., Chyrka, T. \& Maharramov, A. M. (2019a). Acta Cryst. E75, 15441547.

Duruskari, G. S., Khalilov, A. N., Akkurt, M., Mammadova, G. Z., Chyrka, T. \& Maharramov, A. M. (2019b). Acta Cryst. E75, 11751179.

Groom, C. R., Bruno, I. J., Lightfoot, M. P. \& Ward, S. C. (2016). Acta Cryst. B72, 171-179.

Gurbanov, A. V., Maharramov, A. M., Zubkov, F. I., Saifutdinov, A. M. \& Guseinov, F. I. (2018). Aust. J. Chem. 71, 190-194.

Gurbanov, A. V., Mahmudov, K. T., Kopylovich, M. N., Guedes da Silva, F. M., Sutradhar, M., Guseinov, F. I., Zubkov, F. I., Maharramov, A. M. \& Pombeiro, A. J. L. (2017). Dyes Pigments, 138, 107-111.

Hathwar, V. R., Sist, M., Jørgensen, M. R. V., Mamakhel, A. H., Wang, X., Hoffmann, C. M., Sugimoto, K., Overgaard, J. \& Iversen, B. B. (2015). IUCrJ, 2, 563-574.

Khalilov, A. N., Atioğlu, Z., Akkurt, M., Duruskari, G. S., Toze, F. A. A. \& Huseynova, A. T. (2019). Acta Cryst. E75, 662-666. 
Maharramov, A. M., Duruskari, G. Sh., Mammadova, G. Z., Khalilov, A. N., Aslanova, J. M., Cisterna, J., Cárdenas, A. \& Brito, I. (2019). J. Chil. Chem. Soc. 64, 4441-4447.

Maharramov, A. M., Khalilov, A. N., Gurbanov, A. V., Allahverdiyev, M. A. \& Ng, S. W. (2011). Acta Cryst. E67, 0721.

Maharramov, A. M., Shikhaliyev, N. Q., Suleymanova, G. T., Gurbanov, A. V., Babayeva, G. V., Mammadova, G. Z., Zubkov, F. I., Nenajdenko, V. G., Mahmudov, K. T. \& Pombeiro, A. J. L. (2018). Dyes Pigments, 159, 135-141.

Mahmoudi, G., Seth, S. K., Bauzá, A., Zubkov, F. I., Gurbanov, A. V., White, J., Stilinović, V., Doert, Th. \& Frontera, A. (2018c). CrystEngComm, 20, 2812-2821.

Mahmoudi, G., Zangrando, E., Mitoraj, M. P., Gurbanov, A. V., Zubkov, F. I., Moosavifar, M., Konyaeva, I. A., Kirillov, A. M. \& Safin, D. A. (2018a). New J. Chem. 42, 4959-4971.

Mahmoudi, G., Zaręba, J. K., Gurbanov, A. V., Bauzá, A., Zubkov, F. I., Kubicki, M., Stilinović, V., Kinzhybalo, V. \& Frontera, A. (2018b). Eur. J. Inorg. Chem. pp. 4763-4772.

Mahmudov, K. T., Gurbanov, A. V., Guseinov, F. I. \& Guedes da Silva, M. F. C. (2019). Coord. Chem. Rev. 387, 32-46.

Mahmudov, K. T., Kopylovich, M. N., Guedes da Silva, M. F. C. \& Pombeiro, A. J. L. (2017a). Dalton Trans. 46, 10121-10138.

Mahmudov, K. T., Kopylovich, M. N., Guedes da Silva, M. F. C. \& Pombeiro, A. J. L. (2017b). Coord. Chem. Rev. 345, 54-72.

Mahmudov, K. T., Kopylovich, M. N., Maharramov, A. M., Kurbanova, M. M., Gurbanov, A. V. \& Pombeiro, A. J. L. (2014). Coord. Chem. Rev. 265, 1-37.
Mahmudov, K. T., Kopylovich, M. N. \& Pombeiro, A. J. L. (2013). Coord. Chem. Rev. 257, 1244-1281.

Mamedov, I. G., Bayramov, M. R., Salamova, A. E. \& Maharramov, A. M. (2015). Indian J. Chem. 54B, 1518-1527.

Mamedov, I. G., Farzaliyeva, A. E., Mamedova, Y. V., Hasanova, N. N., Bayramov, M. R. \& Maharramov, A. M. (2018). Indian J. Chem. 57B, 1310-1314.

Martem'yanova, N. A., Chunaev, Y. M., Przhiyalgovskaya, N. M., Kurkovskaya, L. N., Filipenko, O. S. \& Aldoshin, S. M. (1993a). Khim. Geterotsikl. Soedin. pp. 415-419.

Martem'yanova, N. A., Chunaev, Y. M., Przhiyalgovskaya, N. M., Kurkovskaya, L. N., Filipenko, O. S. \& Aldoshin, S. M. (1993b). Khim. Geterotsikl. Soedin. pp. 420-425.

Marthi, K., Larsen, M., Ács, M., Bálint, J. \& Fogassy, E. (1995). Acta Chem. Scand. 49, 20-27.

Marthi, K., Larsen, S., Ács, M., Bálint, J. \& Fogassy, E. (1994). Acta Cryst. B50, 762-771.

Pathania, S., Narang, R. K. \& Rawal, R. K. (2019). Eur. J. Med. Chem. 180, 486-508.

Sheldrick, G. M. (2008). Acta Cryst. A64, 112-122.

Sheldrick, G. M. (2015a). Acta Cryst. A71, 3-8.

Sheldrick, G. M. (2015b). Acta Cryst. C71, 3-8.

Spackman, M. A. \& McKinnon, J. J. (2002). CrystEngComm, 4, 378 392.

Spackman, M. \& Jayatilaka, D. (2009). CrystEngComm, 11, 19-32.

Wolff, S. K., Grimwood, D. J., McKinnon, J. J., Turner, M. J., Jayatilaka, D. \& Spackman, M. A. (2012). Crystal Explorer. University of Western Australia. 


\section{supporting information}

Acta Cryst. (2020). E76, 427-431 [https://doi.org/10.1107/S2056989020001899]

Crystal structure and Hirshfeld surface analysis of (E)-3-(benzylideneamino)-5phenylthiazolidin-2-iminium bromide

Gulnara Sh. Duruskari, Mehmet Akkurt, Gunay Z. Mammadova, Taras Chyrka and Abel M.

Maharramov

Computing details

Data collection: APEX2 (Bruker, 2003); cell refinement: SAINT (Bruker, 2003); data reduction: SAINT (Bruker, 2003); program(s) used to solve structure: SHELXT2014 (Sheldrick, 2015a); program(s) used to refine structure: SHELXL2016 (Sheldrick, 2015b); molecular graphics: SHELXTL (Sheldrick, 2008); software used to prepare material for publication: SHELXTL (Sheldrick, 2008).

(E)-3-(Benzylideneamino)-5-phenylthiazolidin-2-iminium bromide

Crystal data

$\mathrm{C}_{16} \mathrm{H}_{16} \mathrm{~N}_{3} \mathrm{~S}^{+} \cdot \mathrm{Br}^{-}$

$M_{r}=362.29$

Monoclinic, $P 2_{1} / c$

$a=12.138(8) \AA$

$b=8.336(5) \AA$

$c=15.872(9) \AA$

$\beta=93.910(16)^{\circ}$

$V=1602.3(17) \AA^{3}$

$Z=4$

\section{Data collection}

Bruker APEXII CCD

diffractometer

$\varphi$ and $\omega$ scans

Absorption correction: multi-scan

(SADABS; Bruker, 2003)

$T_{\min }=0.582, T_{\max }=0.713$

23979 measured reflections

Refinement

Refinement on $F^{2}$

Least-squares matrix: full

$R\left[F^{2}>2 \sigma\left(F^{2}\right)\right]=0.040$

$w R\left(F^{2}\right)=0.111$

$S=1.06$

3314 reflections

190 parameters

12 restraints
$F(000)=736$

$D_{\mathrm{x}}=1.502 \mathrm{Mg} \mathrm{m}^{-3}$

Mo $K \alpha$ radiation, $\lambda=0.71073 \AA$

Cell parameters from 9891 reflections

$\theta=2.8-26.4^{\circ}$

$\mu=2.69 \mathrm{~mm}^{-1}$

$T=296 \mathrm{~K}$

Plate, colourless

$0.21 \times 0.18 \times 0.13 \mathrm{~mm}$

3314 independent reflections

2742 reflections with $I>2 \sigma(I)$

$R_{\text {int }}=0.049$

$\theta_{\max }=26.5^{\circ}, \theta_{\min }=2.6^{\circ}$

$h=-15 \rightarrow 15$

$k=-10 \rightarrow 10$

$l=-19 \rightarrow 18$

Primary atom site location: structure-invariant direct methods

Secondary atom site location: difference Fourier map

Hydrogen site location: mixed

$\mathrm{H}$-atom parameters constrained

$w=1 /\left[\sigma^{2}\left(F_{\mathrm{o}}^{2}\right)+(0.0521 P)^{2}+1.4845 P\right]$

where $P=\left(F_{\mathrm{o}}{ }^{2}+2 F_{\mathrm{c}}{ }^{2}\right) / 3$ 
$(\Delta / \sigma)_{\max }<0.001$

$\Delta \rho_{\max }=0.74 \mathrm{e} \AA^{-3}$

$\Delta \rho_{\min }=-0.60$ e $\AA^{-3}$

Special details

Geometry. All esds (except the esd in the dihedral angle between two 1.s. planes) are estimated using the full covariance matrix. The cell esds are taken into account individually in the estimation of esds in distances, angles and torsion angles; correlations between esds in cell parameters are only used when they are defined by crystal symmetry. An approximate (isotropic) treatment of cell esds is used for estimating esds involving l.s. planes.

Fractional atomic coordinates and isotropic or equivalent isotropic displacement parameters $\left(\hat{A}^{2}\right)$

\begin{tabular}{|c|c|c|c|c|}
\hline & $x$ & $y$ & $z$ & $U_{\text {iso }} * / U_{\text {eq }}$ \\
\hline $\mathrm{Br} 1$ & 0.36371 & 0.40930 & $0.77766(2)$ & $0.05996(15)$ \\
\hline $\mathrm{S} 1$ & $0.27771(6)$ & $0.50993(10)$ & $0.55109(5)$ & $0.0478(2)$ \\
\hline N1 & 0.52247 (19) & 0.7685 & $0.49030(15)$ & $0.0411(5)$ \\
\hline $\mathrm{N} 2$ & 0.42403 (19) & $0.6853(3)$ & $0.48814(15)$ & $0.0438(5)$ \\
\hline N3 & $0.4551(2)$ & $0.6390(3)$ & $0.63168(16)$ & $0.0500(6)$ \\
\hline $\mathrm{H} 3 \mathrm{~A}$ & 0.431514 & 0.589059 & 0.677388 & $0.060 *$ \\
\hline H3B & 0.509254 & 0.711959 & 0.640888 & $0.060 *$ \\
\hline $\mathrm{C} 1$ & $0.3432(2)$ & $0.6653(4)$ & 0.41558 (19) & $0.0467(7)$ \\
\hline $\mathrm{H} 1 \mathrm{~A}$ & 0.380449 & 0.651339 & 0.363896 & $0.056^{*}$ \\
\hline H1B & 0.295261 & 0.758163 & 0.409430 & $0.056^{*}$ \\
\hline $\mathrm{C} 2$ & 0.2763 & $0.5140(4)$ & $0.4348(2)$ & $0.0489(7)$ \\
\hline $\mathrm{H} 2 \mathrm{~A}$ & 0.315568 & 0.419380 & 0.415778 & $0.059 *$ \\
\hline C3 & $0.3971(2)$ & 0.6205 & $0.56063(18)$ & $0.0412(6)$ \\
\hline $\mathrm{C} 4$ & $0.5460(2)$ & $0.8450(4)$ & 0.42487 (19) & $0.0440(6)$ \\
\hline $\mathrm{H} 4 \mathrm{~A}$ & 0.495374 & 0.850348 & 0.378264 & $0.053^{*}$ \\
\hline $\mathrm{C} 5$ & $0.6520(2)$ & $0.9241(3)$ & $0.42299(14)$ & $0.0416(6)$ \\
\hline C6 & $0.7284(2)$ & $0.9221(4)$ & 0.49283 (19) & $0.0513(7)$ \\
\hline H6A & 0.710541 & 0.874346 & 0.543049 & $0.062 *$ \\
\hline $\mathrm{C} 7$ & $0.8319(2)$ & $0.9924(4)$ & $0.4867(2)$ & $0.0663(10)$ \\
\hline $\mathrm{H} 7 \mathrm{~A}$ & 0.883751 & 0.988871 & 0.532539 & $0.080 *$ \\
\hline $\mathrm{C} 8$ & 0.8581 & $1.0679(4)$ & $0.41226(19)$ & $0.0652(10)$ \\
\hline H8A & 0.927072 & 1.114885 & 0.408687 & $0.078^{*}$ \\
\hline C9 & $0.7808(2)$ & $1.0730(4)$ & $0.3432(2)$ & $0.0651(10)$ \\
\hline H9A & 0.797532 & 1.124866 & 0.293831 & $0.078 *$ \\
\hline $\mathrm{C} 10$ & $0.6782(2)$ & 0.9998 (4) & $0.34862(17)$ & $0.0551(8)$ \\
\hline H10A & 0.626928 & 1.001586 & 0.302329 & $0.066^{*}$ \\
\hline $\mathrm{C} 11$ & $0.1609(2)$ & 0.5115 & $0.39571(17)$ & $0.0446(6)$ \\
\hline $\mathrm{C} 12$ & $0.1299(2)$ & $0.3891(4)$ & $0.3387(2)$ & $0.0613(9)$ \\
\hline H12A & 0.180793 & 0.310914 & 0.325946 & $0.074 *$ \\
\hline $\mathrm{C} 13$ & $0.0225(2)$ & $0.3838(5)$ & $0.3009(2)$ & $0.0690(10)$ \\
\hline H13A & 0.002162 & 0.302592 & 0.262799 & $0.083 *$ \\
\hline $\mathrm{C} 14$ & -0.0538 & $0.5001(4)$ & $0.3205(2)$ & $0.0661(10)$ \\
\hline H14A & -0.124515 & 0.498545 & 0.293897 & $0.079 *$ \\
\hline $\mathrm{C} 15$ & -0.0251 & $0.6186(4)$ & $0.3794(2)$ & $0.0683(10)$ \\
\hline H15A & -0.076995 & 0.693888 & 0.394077 & $0.082 *$ \\
\hline $\mathrm{C} 16$ & $0.0820(2)$ & $0.6235(4)$ & $0.4163(2)$ & $0.0631(9)$ \\
\hline
\end{tabular}




\begin{tabular}{|c|c|c|c|c|c|c|}
\hline H16A & 0.1013 & & 3134 & 0.455623 & $0.076^{*}$ & \\
\hline \multicolumn{7}{|c|}{ Atomic displacement parameters $\left(\AA^{2}\right)$} \\
\hline & $U^{11}$ & $U^{22}$ & $U^{33}$ & $U^{12}$ & $U^{13}$ & $U^{23}$ \\
\hline $\mathrm{Br} 1$ & $0.0594(2)$ & $0.0700(3)$ & $0.0489(2)$ & $-0.01088(16)$ & $-0.00776(15)$ & $0.01409(15)$ \\
\hline S1 & $0.0461(4)$ & $0.0511(4)$ & $0.0467(4)$ & $-0.0073(3)$ & $0.0065(3)$ & $0.0068(3)$ \\
\hline N1 & $0.0379(12)$ & $0.0421(13)$ & $0.0432(13)$ & $-0.0028(10)$ & $0.0028(10)$ & $0.0008(10)$ \\
\hline N2 & $0.0387(12)$ & $0.0496(14)$ & $0.0429(13)$ & $-0.0054(10)$ & $0.0013(10)$ & $0.0085(11)$ \\
\hline N3 & $0.0544(15)$ & $0.0552(15)$ & $0.0405(13)$ & $-0.0076(12)$ & $0.0042(11)$ & $0.0037(11)$ \\
\hline $\mathrm{C} 1$ & $0.0426(15)$ & $0.0550(17)$ & $0.0418(15)$ & $-0.0053(13)$ & $-0.0020(12)$ & $0.0096(13)$ \\
\hline $\mathrm{C} 2$ & $0.0471(16)$ & $0.0482(17)$ & $0.0515(17)$ & $-0.0002(13)$ & $0.0038(13)$ & $-0.0012(14)$ \\
\hline $\mathrm{C} 3$ & $0.0406(14)$ & $0.0414(14)$ & $0.0419(15)$ & $0.0029(11)$ & $0.0062(12)$ & $0.0012(12)$ \\
\hline $\mathrm{C} 4$ & $0.0436(15)$ & $0.0431(15)$ & $0.0449(15)$ & $-0.0018(12)$ & $0.0005(12)$ & $0.0055(12)$ \\
\hline $\mathrm{C} 5$ & $0.0422(15)$ & $0.0334(14)$ & $0.0497(16)$ & $-0.0004(11)$ & $0.0063(12)$ & $-0.0022(12)$ \\
\hline C6 & $0.0536(18)$ & $0.0424(16)$ & $0.0566(18)$ & $-0.0077(13)$ & $-0.0055(15)$ & $0.0040(14)$ \\
\hline $\mathrm{C} 7$ & $0.053(2)$ & $0.055(2)$ & $0.087(3)$ & $-0.0103(16)$ & $-0.0162(18)$ & $-0.0012(19)$ \\
\hline $\mathrm{C} 8$ & $0.0486(19)$ & $0.059(2)$ & $0.089(3)$ & $-0.0127(16)$ & $0.0169(19)$ & $-0.0111(19)$ \\
\hline C9 & $0.070(2)$ & $0.068(2)$ & $0.059(2)$ & $-0.0205(19)$ & $0.0244(18)$ & $-0.0054(17)$ \\
\hline $\mathrm{C} 10$ & $0.059(2)$ & $0.060(2)$ & $0.0468(17)$ & $-0.0132(16)$ & $0.0068(15)$ & $-0.0005(15)$ \\
\hline $\mathrm{C} 11$ & $0.0407(15)$ & $0.0474(16)$ & $0.0457(15)$ & $-0.0051(12)$ & $0.0039(12)$ & 0.0059 (13) \\
\hline $\mathrm{C} 12$ & $0.061(2)$ & $0.059(2)$ & $0.065(2)$ & $0.0023(16)$ & $0.0165(17)$ & $-0.0059(17)$ \\
\hline $\mathrm{C} 13$ & $0.063(2)$ & $0.082(3)$ & $0.061(2)$ & $-0.022(2)$ & $0.0058(18)$ & $-0.0193(19)$ \\
\hline $\mathrm{C} 14$ & $0.054(2)$ & $0.083(3)$ & $0.061(2)$ & $-0.0111(19)$ & $0.0019(17)$ & $0.010(2)$ \\
\hline $\mathrm{C} 15$ & $0.055(2)$ & $0.062(2)$ & $0.088(3)$ & $0.0063(17)$ & $0.0043(19)$ & $0.007(2)$ \\
\hline $\mathrm{C} 16$ & $0.060(2)$ & $0.0477(18)$ & $0.083(3)$ & $-0.0009(15)$ & $0.0096(18)$ & $-0.0116(17)$ \\
\hline
\end{tabular}

Geometric parameters ( $\left.\AA,{ }^{\circ}\right)$

\begin{tabular}{llll}
\hline $\mathrm{S} 1-\mathrm{C} 3$ & $1.716(3)$ & $\mathrm{C} 6-\mathrm{H} 6 \mathrm{~A}$ & 0.9300 \\
$\mathrm{~S} 1-\mathrm{C} 2$ & $1.844(3)$ & $\mathrm{C} 7-\mathrm{C} 8$ & $1.394(2)$ \\
$\mathrm{N} 1-\mathrm{C} 4$ & $1.268(4)$ & $\mathrm{C} 7-\mathrm{H} 7 \mathrm{~A}$ & 0.9300 \\
$\mathrm{~N} 1-\mathrm{N} 2$ & $1.380(3)$ & $\mathrm{C} 8-\mathrm{C} 9$ & $1.394(2)$ \\
$\mathrm{N} 2-\mathrm{C} 3$ & $1.332(4)$ & $\mathrm{C} 8-\mathrm{H} 8 \mathrm{~A}$ & 0.9300 \\
$\mathrm{~N} 2-\mathrm{C} 1$ & $1.470(4)$ & $\mathrm{C} 9-\mathrm{C} 10$ & $1.394(2)$ \\
$\mathrm{N} 3-\mathrm{C} 3$ & $1.297(4)$ & $\mathrm{C} 9-\mathrm{H} 9 \mathrm{~A}$ & 0.9300 \\
$\mathrm{~N} 3-\mathrm{H} 3 \mathrm{~A}$ & 0.9000 & $\mathrm{C} 10-\mathrm{H} 10 \mathrm{~A}$ & 0.9300 \\
$\mathrm{~N} 3-\mathrm{H} 3 \mathrm{~B}$ & 0.9001 & $\mathrm{C} 11-\mathrm{C} 16$ & $1.392(2)$ \\
$\mathrm{C} 1-\mathrm{C} 2$ & $1.542(4)$ & $\mathrm{C} 11-\mathrm{C} 12$ & $1.398(2)$ \\
$\mathrm{C} 1-\mathrm{H} 1 \mathrm{~A}$ & 0.9700 & $\mathrm{C} 12-\mathrm{C} 13$ & $1.397(2)$ \\
$\mathrm{C} 1-\mathrm{H} 1 \mathrm{~B}$ & 0.9700 & $\mathrm{C} 12-\mathrm{H} 12 \mathrm{~A}$ & 0.9300 \\
$\mathrm{C} 2-\mathrm{C} 11$ & $1.493(4)$ & $\mathrm{C} 13-\mathrm{C} 14$ & $1.391(2)$ \\
$\mathrm{C} 2-\mathrm{H} 2 \mathrm{~A}$ & 0.9800 & $\mathrm{C} 13-\mathrm{H} 13 \mathrm{~A}$ & 0.9300 \\
$\mathrm{C} 4-\mathrm{C} 5$ & $1.447(4)$ & $\mathrm{C} 14-\mathrm{C} 15$ & $1.389(2)$ \\
$\mathrm{C} 4-\mathrm{H} 4 \mathrm{~A}$ & 0.9300 & $\mathrm{C} 14-\mathrm{H} 14 \mathrm{~A}$ & 0.9300 \\
$\mathrm{C} 5-\mathrm{C} 10$ & $1.3944(19)$ & $\mathrm{C} 15-\mathrm{C} 16$ & $1.390(2)$ \\
$\mathrm{C} 5-\mathrm{C} 6$ & $1.396(2)$ & $\mathrm{C} 15-\mathrm{H} 15 \mathrm{~A}$ & 0.9300 \\
$\mathrm{C} 6-\mathrm{C} 7$ & $1.395(2)$ & $\mathrm{C} 16-\mathrm{H} 16 \mathrm{~A}$ & 0.9300
\end{tabular}




\begin{tabular}{|c|c|c|c|}
\hline $\mathrm{C} 3-\mathrm{S} 1-\mathrm{C} 2$ & 91.65 (14) & $\mathrm{C} 5-\mathrm{C} 6-\mathrm{H} 6 \mathrm{~A}$ & 120.3 \\
\hline $\mathrm{C} 4-\mathrm{N} 1-\mathrm{N} 2$ & $118.4(2)$ & $\mathrm{C} 8-\mathrm{C} 7-\mathrm{C} 6$ & $120.5(3)$ \\
\hline $\mathrm{C} 3-\mathrm{N} 2-\mathrm{N} 1$ & $116.4(2)$ & $\mathrm{C} 8-\mathrm{C} 7-\mathrm{H} 7 \mathrm{~A}$ & 119.8 \\
\hline $\mathrm{C} 3-\mathrm{N} 2-\mathrm{C} 1$ & $116.2(2)$ & $\mathrm{C} 6-\mathrm{C} 7-\mathrm{H} 7 \mathrm{~A}$ & 119.8 \\
\hline $\mathrm{N} 1-\mathrm{N} 2-\mathrm{C} 1$ & $127.4(2)$ & $\mathrm{C} 9-\mathrm{C} 8-\mathrm{C} 7$ & $120.0(3)$ \\
\hline $\mathrm{C} 3-\mathrm{N} 3-\mathrm{H} 3 \mathrm{~A}$ & 117.5 & $\mathrm{C} 9-\mathrm{C} 8-\mathrm{H} 8 \mathrm{~A}$ & 120.0 \\
\hline $\mathrm{C} 3-\mathrm{N} 3-\mathrm{H} 3 \mathrm{~B}$ & 124.6 & $\mathrm{C} 7-\mathrm{C} 8-\mathrm{H} 8 \mathrm{~A}$ & 120.0 \\
\hline $\mathrm{H} 3 \mathrm{~A}-\mathrm{N} 3-\mathrm{H} 3 \mathrm{~B}$ & 116.8 & $\mathrm{C} 8-\mathrm{C} 9-\mathrm{C} 10$ & $119.6(3)$ \\
\hline $\mathrm{N} 2-\mathrm{C} 1-\mathrm{C} 2$ & $105.7(2)$ & $\mathrm{C} 8-\mathrm{C} 9-\mathrm{H} 9 \mathrm{~A}$ & 120.2 \\
\hline $\mathrm{N} 2-\mathrm{C} 1-\mathrm{H} 1 \mathrm{~A}$ & 110.6 & $\mathrm{C} 10-\mathrm{C} 9-\mathrm{H} 9 \mathrm{~A}$ & 120.2 \\
\hline $\mathrm{C} 2-\mathrm{C} 1-\mathrm{H} 1 \mathrm{~A}$ & 110.6 & $\mathrm{C} 9-\mathrm{C} 10-\mathrm{C} 5$ & $120.4(3)$ \\
\hline $\mathrm{N} 2-\mathrm{C} 1-\mathrm{H} 1 \mathrm{~B}$ & 110.6 & $\mathrm{C} 9-\mathrm{C} 10-\mathrm{H} 10 \mathrm{~A}$ & 119.8 \\
\hline $\mathrm{C} 2-\mathrm{C} 1-\mathrm{H} 1 \mathrm{~B}$ & 110.6 & $\mathrm{C} 5-\mathrm{C} 10-\mathrm{H} 10 \mathrm{~A}$ & 119.8 \\
\hline $\mathrm{H} 1 \mathrm{~A}-\mathrm{C} 1-\mathrm{H} 1 \mathrm{~B}$ & 108.7 & $\mathrm{C} 16-\mathrm{C} 11-\mathrm{C} 12$ & $118.8(3)$ \\
\hline $\mathrm{C} 11-\mathrm{C} 2-\mathrm{C} 1$ & $114.9(3)$ & $\mathrm{C} 16-\mathrm{C} 11-\mathrm{C} 2$ & $122.2(2)$ \\
\hline $\mathrm{C} 11-\mathrm{C} 2-\mathrm{S} 1$ & $111.1(2)$ & $\mathrm{C} 12-\mathrm{C} 11-\mathrm{C} 2$ & $118.9(2)$ \\
\hline $\mathrm{C} 1-\mathrm{C} 2-\mathrm{S} 1$ & $104.1(2)$ & $\mathrm{C} 13-\mathrm{C} 12-\mathrm{C} 11$ & $120.2(3)$ \\
\hline $\mathrm{C} 11-\mathrm{C} 2-\mathrm{H} 2 \mathrm{~A}$ & 108.8 & $\mathrm{C} 13-\mathrm{C} 12-\mathrm{H} 12 \mathrm{~A}$ & 119.9 \\
\hline $\mathrm{C} 1-\mathrm{C} 2-\mathrm{H} 2 \mathrm{~A}$ & 108.8 & $\mathrm{C} 11-\mathrm{C} 12-\mathrm{H} 12 \mathrm{~A}$ & 119.9 \\
\hline $\mathrm{S} 1-\mathrm{C} 2-\mathrm{H} 2 \mathrm{~A}$ & 108.8 & $\mathrm{C} 14-\mathrm{C} 13-\mathrm{C} 12$ & $119.9(3)$ \\
\hline $\mathrm{N} 3-\mathrm{C} 3-\mathrm{N} 2$ & $123.5(3)$ & $\mathrm{C} 14-\mathrm{C} 13-\mathrm{H} 13 \mathrm{~A}$ & 120.1 \\
\hline $\mathrm{N} 3-\mathrm{C} 3-\mathrm{S} 1$ & $123.1(2)$ & $\mathrm{C} 12-\mathrm{C} 13-\mathrm{H} 13 \mathrm{~A}$ & 120.1 \\
\hline $\mathrm{N} 2-\mathrm{C} 3-\mathrm{S} 1$ & $113.4(2)$ & $\mathrm{C} 15-\mathrm{C} 14-\mathrm{C} 13$ & $120.4(3)$ \\
\hline $\mathrm{N} 1-\mathrm{C} 4-\mathrm{C} 5$ & $119.7(3)$ & $\mathrm{C} 15-\mathrm{C} 14-\mathrm{H} 14 \mathrm{~A}$ & 119.8 \\
\hline $\mathrm{N} 1-\mathrm{C} 4-\mathrm{H} 4 \mathrm{~A}$ & 120.1 & $\mathrm{C} 13-\mathrm{C} 14-\mathrm{H} 14 \mathrm{~A}$ & 119.8 \\
\hline $\mathrm{C} 5-\mathrm{C} 4-\mathrm{H} 4 \mathrm{~A}$ & 120.1 & $\mathrm{C} 14-\mathrm{C} 15-\mathrm{C} 16$ & $119.3(3)$ \\
\hline $\mathrm{C} 10-\mathrm{C} 5-\mathrm{C} 6$ & $120.0(3)$ & $\mathrm{C} 14-\mathrm{C} 15-\mathrm{H} 15 \mathrm{~A}$ & 120.3 \\
\hline $\mathrm{C} 10-\mathrm{C} 5-\mathrm{C} 4$ & $118.6(2)$ & $\mathrm{C} 16-\mathrm{C} 15-\mathrm{H} 15 \mathrm{~A}$ & 120.3 \\
\hline $\mathrm{C} 6-\mathrm{C} 5-\mathrm{C} 4$ & $121.4(2)$ & $\mathrm{C} 15-\mathrm{C} 16-\mathrm{C} 11$ & $121.3(3)$ \\
\hline $\mathrm{C} 7-\mathrm{C} 6-\mathrm{C} 5$ & $119.5(3)$ & $\mathrm{C} 15-\mathrm{C} 16-\mathrm{H} 16 \mathrm{~A}$ & 119.3 \\
\hline $\mathrm{C} 7-\mathrm{C} 6-\mathrm{H} 6 \mathrm{~A}$ & 120.3 & $\mathrm{C} 11-\mathrm{C} 16-\mathrm{H} 16 \mathrm{~A}$ & 119.3 \\
\hline $\mathrm{C} 4-\mathrm{N} 1-\mathrm{N} 2-\mathrm{C} 3$ & $-173.3(3)$ & $\mathrm{C} 5-\mathrm{C} 6-\mathrm{C} 7-\mathrm{C} 8$ & $1.7(5)$ \\
\hline $\mathrm{C} 4-\mathrm{N} 1-\mathrm{N} 2-\mathrm{C} 1$ & $4.2(4)$ & $\mathrm{C} 6-\mathrm{C} 7-\mathrm{C} 8-\mathrm{C} 9$ & $-0.3(6)$ \\
\hline $\mathrm{C} 3-\mathrm{N} 2-\mathrm{C} 1-\mathrm{C} 2$ & $-24.6(4)$ & $\mathrm{C} 7-\mathrm{C} 8-\mathrm{C} 9-\mathrm{C} 10$ & $-1.1(6)$ \\
\hline $\mathrm{N} 1-\mathrm{N} 2-\mathrm{C} 1-\mathrm{C} 2$ & $157.9(3)$ & $\mathrm{C} 8-\mathrm{C} 9-\mathrm{C} 10-\mathrm{C} 5$ & $1.0(6)$ \\
\hline $\mathrm{N} 2-\mathrm{C} 1-\mathrm{C} 2-\mathrm{C} 11$ & $151.9(3)$ & $\mathrm{C} 6-\mathrm{C} 5-\mathrm{C} 10-\mathrm{C} 9$ & $0.4(5)$ \\
\hline $\mathrm{N} 2-\mathrm{C} 1-\mathrm{C} 2-\mathrm{S} 1$ & $30.1(3)$ & $\mathrm{C} 4-\mathrm{C} 5-\mathrm{C} 10-\mathrm{C} 9$ & $-177.9(3)$ \\
\hline $\mathrm{C} 3-\mathrm{S} 1-\mathrm{C} 2-\mathrm{C} 11$ & $-148.7(2)$ & $\mathrm{C} 1-\mathrm{C} 2-\mathrm{C} 11-\mathrm{C} 16$ & $-63.2(4)$ \\
\hline $\mathrm{C} 3-\mathrm{S} 1-\mathrm{C} 2-\mathrm{C} 1$ & $-24.5(2)$ & $\mathrm{S} 1-\mathrm{C} 2-\mathrm{C} 11-\mathrm{C} 16$ & $54.7(4)$ \\
\hline $\mathrm{N} 1-\mathrm{N} 2-\mathrm{C} 3-\mathrm{N} 3$ & $3.6(4)$ & $\mathrm{C} 1-\mathrm{C} 2-\mathrm{C} 11-\mathrm{C} 12$ & $119.2(3)$ \\
\hline $\mathrm{C} 1-\mathrm{N} 2-\mathrm{C} 3-\mathrm{N} 3$ & $-174.1(3)$ & $\mathrm{S} 1-\mathrm{C} 2-\mathrm{C} 11-\mathrm{C} 12$ & $-122.9(3)$ \\
\hline $\mathrm{N} 1-\mathrm{N} 2-\mathrm{C} 3-\mathrm{S} 1$ & $-176.5(2)$ & $\mathrm{C} 16-\mathrm{C} 11-\mathrm{C} 12-\mathrm{C} 13$ & $2.5(5)$ \\
\hline $\mathrm{C} 1-\mathrm{N} 2-\mathrm{C} 3-\mathrm{S} 1$ & $5.8(3)$ & $\mathrm{C} 2-\mathrm{C} 11-\mathrm{C} 12-\mathrm{C} 13$ & $-179.9(3)$ \\
\hline $\mathrm{C} 2-\mathrm{S} 1-\mathrm{C} 3-\mathrm{N} 3$ & $-168.0(3)$ & $\mathrm{C} 11-\mathrm{C} 12-\mathrm{C} 13-\mathrm{C} 14$ & $-0.4(6)$ \\
\hline $\mathrm{C} 2-\mathrm{S} 1-\mathrm{C} 3-\mathrm{N} 2$ & $12.1(2)$ & $\mathrm{C} 12-\mathrm{C} 13-\mathrm{C} 14-\mathrm{C} 15$ & $-2.1(6)$ \\
\hline $\mathrm{N} 2-\mathrm{N} 1-\mathrm{C} 4-\mathrm{C} 5$ & $-175.7(3)$ & $\mathrm{C} 13-\mathrm{C} 14-\mathrm{C} 15-\mathrm{C} 16$ & $2.5(6)$ \\
\hline
\end{tabular}




$\begin{array}{llll}\mathrm{N} 1-\mathrm{C} 4-\mathrm{C} 5-\mathrm{C} 10 & 176.5(3) & \mathrm{C} 14-\mathrm{C} 15-\mathrm{C} 16-\mathrm{C} 11 & -0.3(6) \\ \mathrm{N} 1-\mathrm{C} 4-\mathrm{C} 5-\mathrm{C} 6 & -1.9(5) & \mathrm{C} 12-\mathrm{C} 11-\mathrm{C} 16-\mathrm{C} 15 & -2.1(6) \\ \mathrm{C} 10-\mathrm{C} 5-\mathrm{C} 6-\mathrm{C} 7 & -1.8(5) & \mathrm{C} 2-\mathrm{C} 11-\mathrm{C} 16-\mathrm{C} 15 & -179.7(3) \\ \mathrm{C} 4-\mathrm{C} 5-\mathrm{C} 6-\mathrm{C} 7 & 176.5(3) & & \end{array}$

Hydrogen-bond geometry $\left(\AA,{ }^{\circ}\right)$

\begin{tabular}{lllll}
\hline$D-\mathrm{H} \cdots A$ & $D-\mathrm{H}$ & $\mathrm{H} \cdots A$ & $D \cdots A$ & $D-\mathrm{H} \cdots A$ \\
\hline $\mathrm{N} 3-\mathrm{H} 3 A \cdots \mathrm{Br} 1$ & 0.90 & 2.37 & $3.258(3)$ & 168 \\
$\mathrm{~N} 3-\mathrm{H} 3 B \cdots \mathrm{Br} 1^{\mathrm{i}}$ & 0.90 & 2.55 & $3.399(3)$ & 158 \\
\hline
\end{tabular}

Symmetry code: (i) $-x+1, y+1 / 2,-z+3 / 2$. 